\title{
Two New Calculating Methods for the Irregular Hermite Interpolation Polynomial
}

\author{
Miao Luo \\ School of Computer Science and Technology \\ Panzhihua University \\ Panzhihua, China \\ e-mail: 4597686@qq.com
}

\author{
Liangliang Ma \\ School of Computer Science and Technology \\ Panzhihua University \\ Panzhihua, China \\ e-mail: mll198684@126.com
}

\begin{abstract}
Based on the theory of the regular Hermite interpolation polynomial, two new calculating methods including undetermined coefficient and Lagrange interpolation have been proposed to solve the complex irregular Hermite interpolation polynomial.
\end{abstract}

Keywords- Hermite interpolation polynomial; undetermined coefficient; Lagrange interpolation

\section{THEORY OF THE HERMITE INTERPOLATION}

Set $y=f(x)$ has definition in interval $[a, b]$, and has corresponding values $y_{0}, y_{1}, \cdots, y_{n}$ on the points of $a \leq x_{0}<x_{1}<\cdots<x_{n} \leq b$, if there is a simple function $P(x)$ satisfied the following formula:

$$
P\left(x_{i}\right)=y_{i} \quad(i=1,2, \cdots, n)
$$

where $P(x)$ is the interpolation function of $f(x), x_{0}, x_{1}$, $\cdots, x_{n}$ are the interpolation nodes, interval $[a, b]$ is the interpolation interval $^{[1]}$.

If $P(x)$ is the polynomial with the number not more than $n$, namely

$$
P(x)=a_{0}+a_{1} x+\cdots+a_{n} x_{n}
$$

where $a_{i}(i=0,1, \cdots, n)$ are the real numbers, we can say $P(x)$ is a interpolation polynomial.

Suppose $y_{j}=f\left(x_{j}\right), m_{j}=f^{\prime}\left(x_{j}\right)(j=0,1, \cdots, n)$ on the nodes $a \leq x_{0}<x_{1}<\cdots<x_{n} \leq b$, if there is an interpolation polynomial $H(x)$ satisfied the conditions (3):

$$
H\left(x_{j}\right)=y_{j}, H^{\prime}\left(x_{j}\right)=m_{j} \quad(j=0,1, \cdots, n)
$$

then $H(x)$ is the Hermite interpolation polynomial ${ }^{[2]}$.

If $y_{i}$ and $m_{j}(i=0,1, \cdots, n)$ are all known on the nods $a \leq x_{0}<x_{1}<\cdots<x_{n} \leq b$, we can say $H(x)$ is the regular Hermite interpolation polynomial, correspondingly, the methods of seeking $H(x)$ is the regular Heremite interpolation ; If only some of $m_{j}(i=0,1, \cdots, n)$ are known on the nodes $a \leq x_{0}<x_{1}<\cdots<x_{n} \leq b$, we can

$\overline{\text { Corresponding author: Liangliang Ma, e-mail: mll198684@126.com }}$ say $H(x)$ is the irregular Hermite interpolation polynomial, the methods of seeking $H(x)$ is the irregular Heremite interpolation $^{[3-4]}$.

\section{TWO PLANS FOR SOLVING THE IRREGULAR HEREMITE} INTERPOLATION POLYNOMIAL

$$
\text { If } y_{i}(i=0,1, \cdots, n) \text { and } m_{j}(j=0,1, \cdots, k ; k<n)
$$

are all known on the points $a \leq x_{0}<x_{1}<\cdots<x_{n} \leq b$, and $m_{j}(j=k+1, \cdots, n)$ are unknown. In this paper, we want to seek an irregular Heremite interpolation polynomial to meet the following equations:

$$
\left\{\begin{array}{l}
H\left(x_{i}\right)=y_{i} \quad(i=0,1, \cdots, n) \\
H^{\prime}\left(x_{j}\right)=m_{j} \quad(j=0,1, \cdots, k ; k<n)
\end{array}\right.
$$

\section{A. Undetermined coefficient}

Bring the known conditions into the irregular Hermite interpolation polynomial, and to determine the required irregular Hermite interpolation polynomial through solving equations to determine its coefficients.

By equations (4), we can know that the equations own $n+k+2$ known conditions. Due to $n+k+2$ known conditions can uniquely determine a polynomial with the number not more than $n+k+1$, namely

$$
H_{n+k+1}(x)=H(x)
$$

so we can suppose the form of $H_{n+k+1}(x)$ as follows:

$$
H_{n+k+1}(x)=a_{0}+a_{1} x+\cdots+a_{n+k+1} x^{n+k+1}
$$

Bring the unknown conditions $H\left(x_{i}\right)=y_{i} \quad(i=0,1, \cdots, n)$ and $H^{\prime}\left(x_{j}\right)=m_{j}(j=0,1, \cdots, k ; k<n)$ into the formula (6), we can get the following equations:

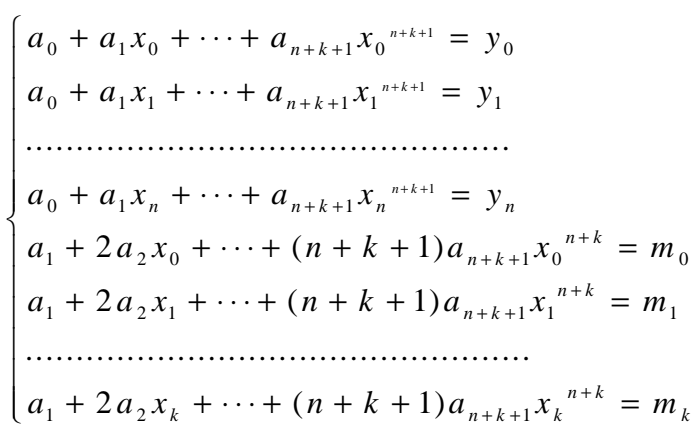


Solving equations (7), we can obtain the coefficients $a_{0}, a_{1}, \cdots, a_{n+k+1}$, and determine $H_{n+k+1}(x)$ finally.

\section{B. Lagrange interpolation}

Using the known conditions $H\left(x_{i}\right)=y_{i} \quad(i=0,1, \cdots, n)$, we can seek a Lagrange interpolation polynomial $L_{n}(x)$. Suppose $H_{n+k+1}(x)$ exists, and bring the known conditions $H\left(x_{i}\right)=y_{i} \quad(i=0,1, \cdots, n)$ and $H^{\prime}\left(x_{j}\right)=m_{j} \quad(j=0,1, \cdots, k ; k<n)$ into $H_{n+k+1}(x)$, and we can determine the required irregular Hermite interpolation polynomial.

Through $H\left(x_{i}\right)=y_{i} \quad(i=0,1, \cdots, n)$, we can seek the Lagrange interpolation polynomial $L_{n}(x)$ :

$$
L_{n}(x)=\sum_{i=0}^{n} y_{i} l_{i}(x)
$$

where

$$
l_{i}(x)=\frac{\left(x-x_{0}\right) \cdots\left(x-x_{i-1}\right)\left(x-x_{i+1}\right) \cdots\left(x-x_{n}\right)}{\left(x_{i}-x_{0}\right) \cdots\left(x_{i}-x_{i-1}\right)\left(x_{i}-x_{i+1}\right) \cdots\left(x_{i}-x_{n}\right)}
$$$$
(i=0,1, \cdots, n)
$$

Due to $l_{i}(x)(i=0,1, \cdots, n)$ satisfy the following conditions on the nodes $x_{0}<x_{1}<\cdots<x_{n}$

$$
l_{i}\left(x_{j}\right)=\left\{\begin{array}{ll}
1, & i=j, \\
0, & i \neq j
\end{array} \quad(i, j=0,1, \cdots, n)\right.
$$

namely $l_{0}(x), l_{1}(x), \cdots, l_{n}(x)$ are the interpolation basis functions on the nodes $x_{0}, x_{1}, \cdots, x_{n}$. By formula (8), we can obtain the expression (11) and (12):

$$
\begin{gathered}
L_{n}\left(x_{j}\right)=\sum_{i=0}^{n} y_{i} l_{i}\left(x_{j}\right)=y_{j}(j=0,1, \cdots, n) \\
L_{n}^{\prime}\left(x_{j}\right)=\sum_{i=0}^{n} y_{i} l_{i}^{\prime}\left(x_{j}\right)=m_{j}(j=0,1, \cdots, n) \\
\text { Suppose } \quad H_{n+k+1}(x)=L_{n}(x)+Q_{n+k+1}(x)
\end{gathered}
$$

Set the highest coefficients of $Q_{n+k+1}(x)$ as 1 , and bring $\left(x_{0}, y_{0}\right)$ into (13), through $L_{n}\left(x_{0}\right)=y_{0}$, we can obtain

$$
Q_{n+k+1}\left(x_{0}\right)=0
$$

namely $x_{0}$ is one of the zero point of $Q_{n+k+1}(x)$. Then bring $\left(x_{1}, y_{1}\right)\left(x_{0}, y_{0}\right)$ into (13), through $L_{n}\left(x_{1}\right)=y_{1}$, we can obtain

$$
Q_{n+k+1}\left(x_{1}\right)=0
$$

namely $x_{1}$ is one of the zero point of $Q_{n+k+1}(x)$. Similarly, bring $\left(x_{2}, y_{2}\right),\left(x_{3}, y_{3}\right), \cdots,\left(x_{n}, y_{n}\right)$ into (13), through $L_{n}\left(x_{j}\right)=\sum_{i=0}^{n} y_{i} l_{i}\left(x_{j}\right)=y_{j} \quad(j=2,3, \cdots, n)$, we can obtain

$$
Q_{n+k+1}\left(x_{j}\right)=0 \quad(j=2,3, \cdots, n)
$$

namely $x_{j}(j=2,3, \cdots, n)$ are the zero points of $Q_{n+k+1}(x)$. Because the number of $Q_{n+k+1}(x)$ is $n+k+1$, so we can suppose

$$
Q_{n+k+1}(x)=\left(x-x_{0}\right)\left(x-x_{1}\right) \cdots\left(x-x_{n}\right) A_{d}(x)
$$

where $A_{d}(x)$ is a polynomial with the number of $d$.

Through $H_{n+k+1}(x)=L_{n}(x)+Q_{n+k+1}(x)$, we can obtain

$$
H^{\prime}{ }_{n+k+1}(x)=L_{n}^{\prime}(x)+Q_{n+k+1}^{\prime}(x)
$$

bring $H^{\prime}\left(x_{j}\right)=m_{j}(j=0,1, \cdots, k ; k<n)$ into (17),

through $L_{n}^{\prime}\left(x_{j}\right)=\sum_{i=0}^{n} y_{i} l_{i}^{\prime}\left(x_{j}\right)=m_{j}(j=0,1, \cdots, k ; k<n)$,

we can obtain

$$
Q_{n+k+1}^{\prime}\left(x_{j}\right)=0(j=0,1, \cdots, k ; k<n)
$$

namely $x_{j}(j=0,1, \cdots, k ; k<n)$ are the double roots of $Q_{n+k+1}(x)$, so

$Q_{n+k+1}(x)=\left(x-x_{0}\right)^{2}\left(x-x_{1}\right)^{2} \cdots\left(x-x_{k}\right)^{2}\left(x-x_{k+1}\right) \cdots$ $\left(x-x_{n}\right) B(x)$

Owning to the number of $Q_{n+k+1}(x)$ is $n+k+1$, and the number of the polynomial

$\left(x-x_{0}\right)^{2}\left(x-x_{1}\right)^{2} \cdots\left(x-x_{k}\right)^{2}\left(x-x_{k+1}\right) \cdots\left(x-x_{n}\right)$ is $n+k+2$, so the number of $B(x)$ is -1 , namely

$$
B(x)=x^{-1}
$$

To sum up, $H_{n+k+1}(x)$ can be uniquely determined by $L_{n}(x)$ and $Q_{n+k+1}(x)$.

\section{REFERENCES}

[1] Yang Shijun, and Wang Xinghua, "Expressions of Hermite interpolation polynomials as divided differences with applications," Appl. Math. J. Chinese Univ. Ser. A, vol. 21, pp. 70-78, January 2006.

[2] Wang Ziyu, Shi Yingguang, Tian Jishan and Liang XueZhang, "Some derelopment and question of polynomials interpolation (1)," Journal of Baoji College of Arts and Sciences (Natural Science), vol. 2, Febuary 1963, pp. 1-5.

[3] Deng Yongchang, "Existence and uniqueness theorem of Hermite interp0Iation polynomial," JournaL of Gansu University of Technology, vol.6, pp.1-5, Febuary 1995.

[4] Li Qignyang, Wang Nengyi, and Yi Dayi, Numerical Analysis. Wuhan: Huazhong University of science and technology press, 2006. 